\title{
On the generation of magnetostatic solutions from gravitational two-soliton solutions of a stationary mass
}

\author{
A. Chaudhuri ${ }^{1, \mathrm{a}}$, S. Chaudhuri ${ }^{2, \mathrm{~b}}$ \\ ${ }^{1}$ Department of Physics, B.K.C. College, 111/2, B.T. Road, Bon Hooghly, Kolkata 700108, India \\ ${ }^{2}$ Department of Physics, University of Burdwan, Golapbag, Burdwan, WB 713104, India
}

Received: 4 September 2017 / Accepted: 20 September 2017 / Published online: 7 November 2017

(C) The Author(s) 2017. This article is an open access publication

\begin{abstract}
In the paper, magnetostatic solutions of the Einstein-Maxwell field equations are generated from the gravitational two-soliton solutions of a stationary mass. Using the soliton technique of Belinskii and Zakharov (Sov Phys JETP 48:985, 1978, Sov Phys JETP 50:1, 1979), we construct diagonal two-soliton solutions of Einstein's gravitational field equations for an axially symmetric stationary space-time and investigate some properties of the generated stationary gravitational metric. Magnetostatic solutions corresponding to the generated stationary gravitational solutions are then constructed using the transformation technique of Das and Chaudhuri (Pramana J Phys 40:277, 1993). The mass and the dipole moment of the source are evaluated. In our analysis we make use of a second transformation (Chaudhuri in Pramana J Phys 58:449, 2002), probably for the first time in the literature, to generate magnetostatic solutions from the stationary gravitational two-soliton solutions which give us simple and straightforward expressions for the mass and the magnetic dipole moment.
\end{abstract}

\section{Introduction}

In the paper, magnetostatic solutions of Einstein-Maxwell field equations are constructed from the stationary twosoliton solutions of Einstein's vacuum field equations in general relativity. For this purpose we first construct diagonal two-soliton solutions for a stationary axially symmetric space-time using the soliton technique of Belinskii and Zakharov [1,2]. The generated stationary gravitational solutions are then used to construct static magnetovac solutions of Einstein-Maxwell field equations. We have also investigated some properties of the generated stationary

\footnotetext{
${ }^{a}$ e-mail: avinandac@gmail.com

b e-mail: schaudhuri.phys@gmail.com
}

gravitational two-soliton metric. The solutions are found to be asymptotically flat containing monopole, dipole and other higher mass multipoles. Under some restrictions on the constants, it is shown that the solutions reduce to the Schwarzschild [3], Kerr [4] and Kerr-NUT [5] metrics. When no such restrictions are imposed, the solutions may be interpreted as the nonlinear superposition of the Kerr metric with an external gravitational field. Our solution possesses an event horizon. The surface area of the horizon is evaluated.

Magnetostatic solutions are then constructed from the generated stationary gravitational two-soliton metric using the simple transformation of Das and Chaudhuri [6]. To relate the metric coefficients obtained by the soliton technique and those of the static magnetovac solutions, we have used a second transformation presented by Chaudhuri [7] by which magnetostatic solutions are obtained in a straightforward way from the gravitational two-soliton solutions.

The mass and the magnetic dipole moment of the source are evaluated. It may be mentioned here that the method followed in the paper to construct magnetostatic solutions deserves attention, although the transformation techniques are already known. The use of the second transformation makes our procedure of obtaining magnetostatic solutions from stationary gravitational two-soliton solutions very simple and probably was not used before in the literature for the purpose. The results are obtained in a compact form. It is expected that this is indeed an addition to the list of new solution generating techniques of Einstein/EinsteinMaxwell field equations.

In Sect. 2, the soliton technique of Belinskii and Zakharov $[1,2]$ is briefly described. In Sect. 3, stationary axially symmetric vacuum solutions using the soliton technique are generated. Magnetostatic solutions corresponding to the generated stationary gravitational solutions are constructed in Sect. 4. Our conclusion follows in Sect. 5. 


\section{The soliton technique}

The soliton technique discovered by Belinskii and Zakharav (BZ) $[1,2]$ is a very useful method of generating exact solutions of Einstein's gravitational field equations. Several authors have presented a number of exact solutions of Einstein's field equations using this technique. Since the method of $\mathrm{BZ}$ is well known in the literature, here we describe the technique in brief. For a detailed study of the soliton technique one may consult references [1,2,8-12].

A general stationary axially symmetric line element in $(r, z)$ coordinates may be represented by

$\mathrm{d} s^{2}=g_{a b} \mathrm{~d} x^{a} \mathrm{~d} x^{b}+e^{\nu}\left(\mathrm{d} r^{2}+\mathrm{d} z^{2}\right)$,

where $a, b$ take values 1,2 and $t, \phi=x^{1}, x^{2}$, respectively. The metric coefficients $g_{a b}$ and $v$ are functions of $r, z$ only.

The Einstein field equations for the metric (1) are [2]

$U_{r}+V_{z}=0$,

$v_{r}=-r^{-1}+(4 r)^{-1} \operatorname{Tr}\left(U^{2}-V^{2}\right)$,

$v_{z}=(2 r)^{-1} \operatorname{Tr}(U V)$

where $U=r g_{r} g^{-1}$ and $V=r g_{z} g^{-1}$

The subscripts $r, z$ denote partial differentiations. The metric coefficient $v$ can be determined from Eqs. (3) and (4) once $g$ is known and the integrability condition of $v$ is Eq. (2). For physically acceptable solutions we have the supplementary condition [9]

det $g_{o}=-r^{2}$,

where $g_{0}$ is a $(2 \times 2)$ matrix associated with $g_{a b}$. Soliton solutions to Eq. (2) are obtained by solving the following two differential equations $[1,2]$ :

$$
\begin{aligned}
& \left(\partial_{r}+\frac{2 \lambda r}{\lambda^{2}+r^{2}} \partial_{\lambda}\right) \Omega=\frac{r U+\lambda V}{\lambda^{2}+r^{2}} \Omega, \\
& \left(\partial_{z}-\frac{2 \lambda^{2}}{\lambda^{2}+r^{2}} \partial_{\lambda}\right) \Omega=\frac{r V-\lambda U}{\lambda^{2}+r^{2}} \Omega,
\end{aligned}
$$

where $\lambda$ is a complex spectral parameter and the eigenfunction $\Omega$ is a two-dimensional matrix which satisfies the relation

$\left.\Omega(r, z, \lambda)\right|_{\lambda=0}=g_{0}(r, z)$.

Once $\Omega$ is known, the generated metric coefficients are obtained from the following relations $[2,9,12]$ :

$g_{a b}^{\prime}=\left(g_{o}\right)_{a b}-\sum_{k, l=1}^{N} N_{a}^{l}\left(\Gamma^{-1}\right)_{l k} N_{b}^{k} \mu_{k}^{-1} \mu_{l}^{-1}$,
$\Gamma_{k l}=m_{c}^{k}\left(g_{o}\right)_{c a} m_{a}^{l}\left(\mu_{k} \mu_{l}+r^{2}\right)^{-1}$,

$m_{a}^{k}=m_{o c}^{k}\left[\Omega^{-1}\left(r, z, \lambda=\mu_{k}\right)\right]_{c a}$,

$N_{a}^{k}=m_{b}^{k}\left(g_{o}\right)_{b a}$,

$\mu_{k}=\omega_{k}-z+\left[\left(\omega_{k}-z\right)^{2}+r^{2}\right]^{1 / 2}$,

where $m_{o c}^{k}$ and $\omega_{k}$ are arbitrary constants and $\mu_{k}$ 's are the pole trajectories. The indices $k$ and $l$ can take values from 1 to $N$. Here $N$ is the number of solitons i.e. the number of poles that appears in the scattering matrix. For $N=1$, it is called one-soliton solutions, $N=2$, is the two-soliton solutions and so on.

The new metric $g_{a b}^{\prime}$ given by Eq. (10) is a solution to Eq. (2) but, in general, it does not satisfy Eq. (6). A physically acceptable $g=g_{a b}^{p h}$ is defined by the relation [9]

$g_{a b}^{p h}=-r\left(-\operatorname{det} g^{\prime}\right)^{-1 / 2} g_{a b}^{\prime}$,

where, $\quad \operatorname{det} g^{\prime}=(-1)^{N} r^{2 N}\left(\prod_{k=1}^{N}\left(\mu_{k}^{2}\right)^{-1}\right) \operatorname{det} g_{o}$.

For general $N$-soliton solutions the function $v$ is given by $[9,12]$

$$
\begin{aligned}
v_{N}= & v_{0}+\ln \left[r^{-N^{2} / 2}\right. \\
& \times\left(\prod_{k=1}^{N} \mu_{k}\right)^{N+1}\left(\prod_{\substack{k, l=1 \\
k>l}}^{N}\left(\mu_{k}-\mu_{l}\right)^{2}\right)^{-2} \\
& \left.\times \operatorname{det} \Gamma_{k l}\right]+\ln C_{N},
\end{aligned}
$$

where $C_{N}$ are arbitrary constants and $\nu_{0}$ is the $\nu$-function of the static metric. The expression for $\nu_{0}$ is given in Sect. 3 .

\section{The generated stationary gravitational solutions}

In this section, two-soliton solutions of Einstein's vacuum field equations for an axially symmetric stationary metric are constructed using a Laplace's solution as seed. The solutions are found to be asymptotically flat and contain monopole, dipole and other higher mass multipoles.

We consider a general static axially symmetric metric

$\mathrm{d} s^{2}=e^{\nu_{0}}\left(\mathrm{~d} r^{2}+\mathrm{d} z^{2}\right)+r^{2} e^{-\psi} \mathrm{d} \phi^{2}-e^{\psi} \mathrm{d} t^{2}$,

where $\psi=\psi(r, z)$ is a Laplace solution and $v_{0}$, the $v$ function of the static metric, is given by [9] 
$\nu_{0}(\psi)=-\psi+\frac{1}{2} \int\left[r\left\{\left(\psi_{r}^{2}-\psi_{z}^{2}\right) \mathrm{d} r+2 \psi_{r} \psi_{z} \mathrm{~d} z\right\}\right]$,

where the subscripts $r$ and $z$ denote partial differentiations.

We take Laplace's solution $\psi$ as seed in the general static metric and assume $g_{0}$ as

$g_{o}=\left(\begin{array}{ll}-e^{\psi} & 0 \\ 0 & r^{2} e^{-\psi}\end{array}\right)$

satisfying Eq. (6).

Since the seed solution is diagonal, we take the eigenfunction $\Omega$ as a $2 \times 2$ diagonal matrix and write $\Omega$ in the form

$\Omega=\left(\begin{array}{lc}-e^{F_{k}} & 0 \\ 0 & \left(r^{2}-2 \lambda z-\lambda^{2}\right) e^{-F_{k}}\end{array}\right)$,

such that $F_{k}=\psi$ when $\lambda=0$.

$$
\begin{aligned}
& \mu_{1}=K(x+1)(1-y), \\
& \mu_{2}=K(x-1)(1-y) .
\end{aligned}
$$

With $\psi$ given by Eq. (24), $F_{1}$ and $F_{2}$, in prolate spheroidal coordinates, are obtained:

$$
F_{1}=-\frac{1}{2} \alpha_{0}\left(x^{3}-x y-x+y-y^{3}\right)\left(x^{2}+y^{2}-1\right)^{-\frac{3}{2}},
$$

$F_{2}=-\frac{1}{2} \alpha_{0}\left(x^{3}-x y-x-y+y^{3}\right)\left(x^{2}+y^{2}-1\right)^{-\frac{3}{2}}$.

Using Eqs. (10)-(17) and after cumbersome mathematical calculations, physically acceptable metric coefficients $g_{a b}^{p h}$ are found to be

$$
\begin{aligned}
g_{11}^{p h}= & -\exp \left[\alpha_{0} x y\left(x^{2}+y^{2}-1\right)^{-\frac{3}{2}}\right]\left[\begin{array}{l}
c_{1}^{2}\left(1-y^{2}\right) e^{-2 f_{1}}+c_{2}^{2}\left(1-y^{2}\right) e^{2 f_{1}}-c_{3}^{2}\left(x^{2}-1\right) e^{-2 f_{2}} \\
-c_{4}^{2}\left(x^{2}-1\right) e^{2 f_{2}}+2 c_{1} c_{2}\left(x^{2}-y^{2}\right)
\end{array}\right] D^{-1}, \\
g_{22}^{p h}= & K^{2}\left(x^{2}-1\right)\left(1-y^{2}\right) \exp \left[\alpha_{0} x y\left(x^{2}+y^{2}-1\right)^{-\frac{3}{2}}\right] \\
& \times\left[\begin{array}{l}
c_{1}^{2}(1+y)^{3}(1-y)^{-1} e^{-2 f_{1}}+c_{2}^{2}(1-y)^{3}(1+y)^{-1} e^{2 f_{1}}-c_{3}^{2}(x-1)^{3}(x+1)^{-1} e^{2 f_{2}} \\
-c_{4}^{2}(x+1)^{3}(x-1)^{-1} e^{2 f_{2}}+2 c_{1} c_{2}\left(x^{2}-y^{2}\right)
\end{array}\right] D^{-1}, \\
g_{12}^{p h}= & g_{21}^{p h}=2 K D^{-1}\left[\begin{array}{l}
(x+y)\left\{c_{1} c_{3}(x-1)(1+y) e^{2 u}-c_{2} c_{4}(x+1)(1-y) e^{-2 u}\right\} \\
-(x-y)\left\{c_{1} c_{4}(x+1)(1+y) e^{2 v}-c_{2} c_{3}(x-1)(1-y) e^{-2 v}\right\}
\end{array}\right],
\end{aligned}
$$

The function $F_{k}=F_{k}(r, z, \lambda)$ satisfies the following relations [9]:

$$
\begin{aligned}
& \left(r \frac{\partial}{\partial r}-\lambda \frac{\partial}{\partial z}+2 \lambda \frac{\partial}{\partial \lambda}\right) F_{k}=r \frac{\partial \psi}{\partial r} \\
& \left(r \frac{\partial}{\partial \lambda}+\lambda \frac{\partial}{\partial r}\right) F_{k}=r \frac{\partial \psi}{\partial z} .
\end{aligned}
$$

For two-soliton solutions there are two poles $\mu_{1}$ and $\mu_{2}$. Since the solutions are required along the pole trajectories $\lambda=\mu_{k}, F_{k}$, should have two values, $F_{1}$ and $F_{2}$.

In prolate spheroidal coordinates $(x, y)$ defined by

$r^{2}=K^{2}\left(x^{2}-1\right)\left(1-y^{2}\right), \quad z=z_{1}+K x y$,

we take the Laplace solution $\psi$ in the form

$$
\psi=\alpha_{0} x y\left(x^{2}+y^{2}-1\right)^{-\frac{3}{2}}
$$

where $K, z_{1}$ and $\alpha_{0}$ are constants.

Assuming that the arbitrary constant $\omega_{k}$ appearing in Eq. (14) is real having values $\omega_{1}$ and $\omega_{2}$ for two-soliton solutions, the poles $\mu_{1}$ and $\mu_{2}$ in $(x, y)$ coordinates are found to be

$$
\begin{aligned}
\nu_{2}= & v_{0}+\ln D-\ln \left[16 K^{2}\left(x^{2}-y^{2}\right)\right]+\ln C_{2}, \\
v_{0}= & -\frac{1}{4} \alpha_{0}\left(x^{2}+y^{2}-1\right)^{-\frac{3}{2}}\left[4 x y-\alpha_{0}\left\{\left(x^{2}+y^{2}-1\right)^{2}\right.\right. \\
& \left.\left.-10 x^{2} y^{2}\left(x^{2}+y^{2}-1\right)+9 x^{4} y^{4}\right\}\left(x^{2}+y^{2}-1\right)^{-\frac{5}{2}}\right],
\end{aligned}
$$

$D=c_{1}^{2}(1+y)^{2} e^{-2 f_{1}}+c_{2}^{2}(1-y)^{2} e^{2 f_{1}}+c_{3}^{2}(x-1)^{2} e^{-2 f_{2}}$

$$
+c_{4}^{2}(x+1)^{2} e^{2 f_{2}}-2 c_{1} c_{2}\left(x^{2}-y^{2}\right) \text {, }
$$

$f_{1}=\alpha_{0} x\left(x^{2}-1\right)\left(x^{2}+y^{2}-1\right)^{-\frac{3}{2}}$,

$f_{2}=\alpha_{0} y\left(1-y^{2}\right)\left(x^{2}+y^{2}-1\right)^{-\frac{3}{2}}$,

$2 u=-\left(f_{1}+f_{2}\right)$,

$2 v=\left(f_{1}+f_{2}\right)$,

$c_{1}=m_{02}^{1} m_{02}^{2}\left(4 \omega_{1} \omega_{2}\right)^{-1}, \quad c_{2}=m_{01}^{1} m_{01}^{2}$

$c_{3}=-m_{02}^{1} m_{01}^{2}\left(2 \omega_{1}\right)^{-1}, \quad c_{4}=-m_{02}^{2} m_{01}^{1}\left(2 \omega_{2}\right)^{-1}$.

The stationary metric (1) is thus completely given by Eqs. (28)-(37). 
The asymptotic expansion of $g_{11}^{p h}$ is obtained:

$g_{11}^{p h}=1+\frac{2 a_{2}}{a_{1}} \cdot \frac{1}{x}+\frac{a_{3}}{a_{1}^{2}} \cdot \frac{1}{x^{2}}+\cdots$

where

$$
\begin{aligned}
a_{1}= & \left(c_{3}-c_{4}\right)^{2}, \quad a_{2}=c_{3}^{2}-c_{4}^{2}, \quad a_{3}=a_{4}-a_{1}^{2}\left(1-\alpha_{0} y\right), \\
a_{4}= & 4 a_{2}^{2}-a_{1}\left[a_{5}+2 y\left(c_{1}^{2} \beta_{1}-c_{2}^{2} \beta_{2}\right)\right. \\
& \left.+y^{2}\left(c_{1}^{2} \beta_{1}+c_{2}^{2} \beta_{2}-2 c_{1} c_{2}\right)\right], \\
a_{5}= & c_{1}^{2} \beta_{1}+c_{2}^{2} \beta_{2}+c_{3}^{2}+c_{4}^{2}, \\
\beta_{1}= & 1+2 \alpha_{0}\left(1+\alpha_{0}\right), \quad \beta_{2}=1-2 \alpha_{0}\left(1-\alpha_{0}\right) .
\end{aligned}
$$

The static limit of the solutions (28)-(30) is obtained with

$m_{01}^{2}=0, \quad m_{02}^{1}=0$.

In the static limit one obtains

$g_{11}^{p h}=\left(\frac{x-1}{x+1}\right) \exp \left[\alpha_{0} x y\left(x^{2}+y^{2}-1\right)^{-\frac{3}{2}}\right]$.

This may be interpreted as the Schwarzschild metric embedded in an external gravitational field. When the parameter $\alpha_{0}$, representing the strength of the external field, vanishes, i.e. $\alpha_{0}=0$, one recovers the Schwarzschild solution [3]

$g_{11}^{p h}(S c h)=\left(\frac{x-1}{x+1}\right)$.

If we redefine the constants by

$c_{3}-c_{4}=k, \quad c_{3}+c_{4}=-m, \quad c_{1}+c_{2}=a, \quad c_{1}-c_{2}=h$,

and substitute $\alpha_{0}=0$ in Eq. (28), we obtain

$g_{11}^{p h}=\frac{k^{2} x^{2}+a^{2} y^{2}-\left(m^{2}+h^{2}\right)}{(k x+m)^{2}+(a y+h)^{2}}$.

Equation (43) is the Kerr-NUT solution [5] with NUT parameter $h$. Its reduction to the Kerr metric [4] is only a trivial task and can be obtained by substituting $h=0$,

$g_{11}^{p h}($ Kerr $)=\frac{p^{2} x^{2}+q^{2} y^{2}-1}{(p x+1)^{2}+q^{2} y^{2}}$,

where $p$ and $q$ are defined by

$p=\frac{k}{m}, \quad q=\frac{a}{m}, \quad$ and $\quad p^{2}+q^{2}=1$.

The generated solutions presented in Eqs. (28)-(33) may thus be interpreted as the nonlinear superposition of a Kerr metric with an external gravitational field. The parameter $\alpha_{0}$ is related to the strength of the external gravitational field.
The event horizon of the constructed stationary metric is at $x=x_{h}=1$. The infinite red shift surface (i.r.s.) depends on the values of $y$ i.e. $x_{\text {i.r.s. }}=x(y)$ and in general, $x_{\text {i.r.s. }}>x_{h}$. At the poles $(y= \pm 1)$, these two surfaces coincide. The event horizon is thus always surrounded by the infinite red shift surface similar to the Kerr metric.

The surface area of the event horizon is found to be

$S=16 \pi K^{2} c_{4}\left(c_{4}-c_{3}\right)^{-1} \exp \left(-\alpha_{0}\right)$.

In the absence of any external field, if one substitutes $c_{3}=$ 0 and $K=m$, one obtains the area of the event horizon corresponding to the Schwarzschild metric.

\section{Static magnetic dipole solution}

The static magnetic dipole solution corresponding to the generated two-soliton stationary metric (28)-(33) may be obtained using the technique of Das and Chaudhuri [6]. In the following we describe in brief the technique of Das and Chaudhuri to generate magnetostatic solutions from stationary gravitational fields.

In cylindrical coordinates $(r, z)$, an axially symmetric static line element is represented by [13]

$\mathrm{d} s^{2}=e^{2 \beta} \mathrm{d} t^{2}-e^{-2 \beta}\left[e^{2 \gamma^{m}}\left(\mathrm{~d} r^{2}+\mathrm{d} z^{2}\right)+r^{2} \mathrm{~d} \phi^{2}\right]$,

where the metric coefficients $\beta$ and $\gamma^{m}$ are functions of $r, z$ only.

The Einstein-Maxwell field equations corresponding to the metric (47) can be written as $[13,14]$

$\beta, r r+r^{-1} \beta, r+\beta,{ }_{z z}=e^{2 \beta} r^{-2}\left(A_{3, r}^{2}+A_{3, z}^{2}\right)$,

$A_{3, r r}-r^{-1} A_{3, r}^{+} A_{3, z z}=-2\left(\beta, r A_{3, r}+\beta,{ }_{z} A_{3, z}\right)$,

$\gamma,{ }_{r}^{m}=r\left(\beta,{ }_{r}^{2}-\beta, \underset{z}{2}\right)+e^{2 \beta} r^{-2}\left(A_{3, r}^{2}-A_{3, z}^{2}\right)$,

$\gamma_{z}^{m}=2 r\left[\beta, r \beta, z+e^{2 \beta} r^{-2} A_{3, r} A_{3, z}\right]$,

where the comma represents partial differentiation, $A_{3}$ is the true magnetic component of the electromagnetic 4-potential and is related to the pseudo magnetic potential $A_{3}^{\prime}$ by [14]

$A_{3, r}^{\prime}=r^{-1} e^{2 \beta} A_{3, z}$,

$A_{3, z}^{\prime}=-r^{-1} e^{2 \beta} A_{3, r}$.

Now consider an axially symmetric stationary metric in $(r, z)$ coordinates [13],

$\mathrm{d} s^{2}=e^{u}(\mathrm{~d} t-\omega \mathrm{d} \phi)^{2}-e^{-u}\left[e^{2 \gamma^{s}}\left(\mathrm{~d} r^{2}+\mathrm{d} z^{2}\right)+r^{2} \mathrm{~d} \phi^{2}\right]$. 
The metric coefficients $u, \omega$ and $\gamma^{s}$ are functions of $r$ and $z$ only. The vacuum Einstein's field equations are $[6,13]$

$$
\begin{aligned}
& u, r r+u, z z+r^{-1} u, r=-e^{2 u} r^{-2}\left(\omega,{ }_{r}^{2}+\omega, \underset{z}{2}\right) \text {, } \\
& \omega,_{r r}+\omega,_{z z}-r^{-1} \omega,_{r}=-2\left(u,_{r}^{\omega},_{r}+u,,_{z} \omega,{ }_{z}\right) \text {, } \\
& 4 \gamma,{ }_{r}^{s}=r\left(u,{ }_{r}^{2}-u,{ }_{z}^{2}\right)-e^{2 u} r^{-2}\left(\omega,{ }_{r}^{2}-\omega, \underset{z}{2}\right), \\
& 2 \gamma,{ }_{z}^{s}=r\left[u, r u, z-e^{2 u} r^{-2} \omega, r \omega, z\right] .
\end{aligned}
$$

The superscripts $m$ and $s$ refer to the magnetovac and stationary cases, respectively.

Comparing the magnetostatic field equations (48)-(51) of the metric (47) with the stationary gravitational field equations (54)-(57) corresponding to the metric (53), one readily finds that static magnetovac solutions may be generated from the stationary axisymmetric vacuum solutions and vice versa via the transformations

$\beta=u, \quad A_{3}=i \omega, \quad \gamma^{m}=4 \gamma^{s}$.

To obtain physically acceptable solutions, the imaginary quantity $i$ in Eq. (58) is eliminated by the parameter change technique of Das and Banerji $[15,16]$. The generation of magnetostatic solutions from stationary gravitational fields is thus complete.

From Eq. (58), it appears that once $\omega$ is known, $A_{3}$ can be evaluated. Since the metric (1) does not contain any term involving $\omega$, it is very difficult to evaluate $\omega$ directly from $f=-g_{11}$,

$\omega=-\left(2 g_{11}\right)^{-1}\left(g_{12}+g_{21}\right)$.

For a diagonal metric, $\left(\Omega_{0}\right)_{12}=\left(\Omega_{0}\right)_{21}$, and we have $g_{12}=$ $g_{21}$. One thus obtains

$\omega=-g_{12}\left(g_{11}\right)^{-1}$

and

$g_{12}=g_{21}=f \omega$.

The metric coefficient $g_{22}$ is found to be

$g_{22}=K^{2} f^{-1}\left(x^{2}-1\right)\left(1-y^{2}\right)-f \omega^{2}$.

From Eqs. (60), (63), (64) and under the coordinate transformation (23), it is easy to show that det $g_{0}=-r^{2}$. This satisfies the supplementary condition (6).

From Eq. (60) and considering the line elements (53) and (59) we obtain the expression for $e^{u}$ :

$$
\begin{aligned}
e^{u} & =\exp \left[\alpha_{0} x y\left(x^{2}+y^{2}-1\right)^{-\frac{3}{2}}\right] \\
& \times\left[\begin{array}{l}
c_{1}^{2}\left(1-y^{2}\right) e^{-2 f_{1}}+c_{2}^{2}\left(1-y^{2}\right) e^{2 f_{1}}-c_{3}^{2}\left(x^{2}-1\right) e^{-2 f_{2}} \\
-c_{4}^{2}\left(x^{2}-1\right) e^{2 f_{2}}+2 c_{1} c_{2}\left(x^{2}-y^{2}\right)
\end{array}\right] D^{-1} .
\end{aligned}
$$

The other metric coefficient $\omega$ is obtained from Eq. (62) and is found to be

$$
\begin{array}{rl}
\omega=2 & K e^{-\psi} \\
\times & {\left[\begin{array}{l}
(x+y)\left\{c_{1} c_{3}(x-1)(1+y) e^{2 p}-c_{2} c_{4}(x+1)(1-y) e^{-2 p}\right\}-(x-y)\left\{c_{1} c_{4}(x+1)(1+y) e^{2 q}\right. \\
\left.-c_{2} c_{3}(x-1)(1-y) e^{-2 q}\right\}
\end{array}\right]} \\
{\left[c_{1}^{2}\left(1-y^{2}\right) e^{-2 f_{1}}+c_{2}^{2}\left(1-y^{2}\right) e^{2 f_{1}}-c_{3}^{2}\left(x^{2}-1\right) e^{-2 f_{2}}-c_{4}^{2}\left(x^{2}-1\right) e^{2 f_{2}}+2 c_{1} c_{2}\left(x^{2}-y^{2}\right)\right]}
\end{array},
$$

the soliton solutions of Belinskii and Zakharov. However, one can find $\omega$ from the solutions of BZ as follows.

We consider a stationary metric

$$
\mathrm{d} s^{2}=f^{-1}\left[e^{2 \gamma^{s}}\left(\mathrm{~d} r^{2}+\mathrm{d} z^{2}\right)+r^{2} \mathrm{~d} \phi^{2}\right]-f(\mathrm{~d} t-\omega \mathrm{d} \phi)^{2},
$$

where $f, \omega$ and $\gamma^{s}$ are the metric coefficients.

Chaudhuri has shown that the metric coefficients $f$ and $\omega$ for the line element (59) are related to the metric coefficients $g_{a b}$ of the metric (1) by [7] where $D$ is given by Eq. (33).

In order to get real magnetostatic solutions we make the substitutions $c_{1 \rightarrow i c_{1}}$ and $c_{2} \rightarrow i c_{2}$ in Eqs. (65) and (66), and using the transformation (58) we obtain

$$
\begin{aligned}
e^{2 \beta} & =\exp \left[\alpha_{0} x y\left(x^{2}+y^{2}-1\right)^{-3}\right] \\
& \times\left[\begin{array}{l}
c_{1}^{2}\left(1-y^{2}\right) e^{-2 f_{1}}+c_{2}^{2}\left(1-y^{2}\right) e^{2 f_{1}}+c_{3}^{2}\left(x^{2}-1\right) e^{-2 f_{2}} \\
+c_{4}^{2}\left(x^{2}-1\right) e^{2 f_{2}}+2 c_{1} c_{2}\left(x^{2}-y^{2}\right)
\end{array}\right]^{2} D_{1}^{-2},
\end{aligned}
$$




$$
\begin{aligned}
A_{3}= & 2 K e^{-\psi} \\
& \times \frac{\left[\begin{array}{l}
(x+y)\left\{c_{1} c_{3}(x-1)(1+y) e^{2 p}-c_{2} c_{4}(x+1)(1-y) e^{-2 p}\right\}-(x-y)\left\{c_{1} c_{4}(x+1)(1+y) e^{2 q}\right] \\
\left.-c_{2} c_{3}(x-1)(1-y) e^{-2 q}\right\}
\end{array}\right]}{\left[\left\{c_{1}^{2}\left(1-y^{2}\right) e^{-2 f_{1}}+c_{2}^{2}\left(1-y^{2}\right) e^{2 f_{1}}\right\}+\left\{c_{3}^{2}\left(x^{2}-1\right) e^{-2 f_{2}}+c_{4}^{2}\left(x^{2}-1\right) e^{2 f_{2}}+2 c_{1} c_{2}\left(x^{2}-y^{2}\right)\right\}\right]},
\end{aligned}
$$

where

$$
\begin{aligned}
& D_{1}=-\left[c_{1}^{2}(1+y)^{2} e^{-2 f_{1}}+c_{2}^{2}(1-y)^{2} e^{2 f_{1}}\right. \\
& \left.\quad-c_{3}^{2}(x-1)^{2} e^{-2 f_{2}}-c_{4}^{2}(x+1)^{2} e^{2 f_{2}}-2 c_{1} c_{2}\left(x^{2}-y^{2}\right)\right] .
\end{aligned}
$$

The asymptotic expansions of $e^{2 \beta}$ and $A_{3}$ are found to be

$$
\begin{aligned}
e^{2 \beta}= & 1-\frac{4 n_{1}}{x}+\frac{2 n_{2}}{x^{2}}+\cdots \\
A_{3}= & 2 K n_{5}\left[\frac{\left(1-y^{2}\right)}{n_{1} x}-\frac{1}{2}\left\{n_{6} y+n_{7}\left(1-y^{2}\right)\right.\right. \\
& \left.\left.-2 \alpha_{0}\left(n_{8}+3 y\right) y^{2}\right\} \cdot \frac{1}{x^{2}}+\cdots\right],
\end{aligned}
$$

where

$$
\begin{aligned}
& n_{1}=\frac{\left(c_{4}-c_{3}\right)}{\left(c_{4}+c_{3}\right)}, \quad n_{2}=n_{3}+n_{4}, \\
& n_{3}=2\left[\left(c_{1}-c_{2}\right)^{2}+\left(c_{4}-c_{3}\right)^{2}\right]\left(c_{4}+c_{3}\right)^{-2}, \\
& n_{4}=\left[4\left(c_{1}^{2}-c_{2}^{2}\right)\left(c_{4}+c_{3}\right)^{-2}+\alpha_{0}\right] y, \\
& n_{5}=\left(c_{1}+c_{2}\right)\left(c_{3}-c_{4}\right)\left(c_{3}+c_{4}\right)^{-2}, \\
& n_{6}=3 \alpha_{0}+4 n_{8}, \\
& n_{7}=\alpha_{0} n_{8}+n_{9}-2, \\
& n_{8}=\left(\frac{c_{1}-c_{2}}{c_{1}+c_{2}}\right), \\
& n_{9}^{=}\left(\frac{c_{1}+c_{2}}{c_{4}+c_{3}}\right)^{2} .
\end{aligned}
$$

The solutions are thus asymptotically flat at spatial infinity. In Boyer-Lindquist coordinates $(R, \theta)$ defined by

$K x=R-m$, and $y=\cos \theta$,

the asymptotic expansions of $e^{2 \beta}$ and $A_{3}$ are obtained:

$$
e^{2 \beta}=1-\frac{4 K n_{1}}{R}+2 K\left(K n_{2}-2 m n_{1}\right) \cdot \frac{1}{R^{2}}+\cdots
$$

$$
\begin{aligned}
A_{3}= & 2 K n_{5}\left[\frac{K \cos ^{2} \theta}{n_{1} R}\right] \\
& +\frac{1}{2}\left\{K n_{6}+K\left(\frac{2 K m}{n_{1}}+K n_{7}\right) \cos \theta\right. \\
& \left.-2 \alpha_{0} K\left(n_{8}+3 \cos \theta\right) \cos \theta\right\}+\cdots
\end{aligned}
$$

From the asymptotic expansions of $e^{2 \beta}$ and $A_{3}$ (i.e. from Eqs. (74) and (75)), the mass $(M)$ and the dipole moment $(\mu)$ of the source are found to be

$$
\begin{aligned}
& M=2 K \frac{\left(c_{4}-c_{3}\right)}{\left(c_{4}+c_{3}\right)}, \\
& \mu=2 K^{2} \frac{\left(c_{1}+c_{2}\right)}{\left(c_{4}+c_{3}\right)} .
\end{aligned}
$$

If one substitutes $c_{1}=c_{2}=\sqrt{c_{3}}=\alpha$ (here $\alpha$ is any parameter) and $c_{4}=1$ then from Eqs. (76) and (77)

$M=2 K \frac{\left(1-\alpha^{2}\right)}{\left(1+\alpha^{2}\right)}$,

$\mu=4 K^{2} \frac{\alpha}{\left(1+\alpha^{2}\right)}$.

It follows that, if $\alpha=0$, the dipole moment vanishes. One can thus identify $\alpha$ as the magnetic parameter.

If we set $\alpha=1$, it is evident from Eqs. (78) and (79) that $M=0$ and $\mu=2 K^{2}$. One thus obtains the dipole moment of a massless source.

The dipole moment to mass ratio is obtained:

$$
\frac{\mu}{M}=\frac{2 K \alpha}{\left(1-\alpha^{2}\right)} .
$$

In the absence of the magnetic parameter i.e. for $\alpha=0$, if we put $K=m$, we have $M=2 m$. This is not the Schwarzschild solution, but it refers to a mass with monopole moment $2 \mathrm{~m}$. Bonnor [17] constructed a similar solution having the same monopole moment $M=2 m$.

\section{Conclusion}

The solutions (28)-(36) presented in the paper describe a Kerr body embedded in an external gravitational field. Under some restrictions on the constants, the solutions reduce to 
the Schwarzschild, Kerr and Kerr-NUT metrics. The constructed solutions are well behaved at spatial infinity containing monopole, dipole and other higher mass multipole moments. The area of the event horizon is also evaluated.

Magnetostatic solutions corresponding to the stationary gravitational solutions (28)-(36) are constructed in Eqs. (67)-(69). The asymptotic behaviour of the solutions is also discussed. In the absence of a magnetic field the solutions do not correspond to the Schwarzschild metric in a pure vacuum limit; rather one obtains a mass monopole term $M=2 m$. Bonnor also presented a magnetostatic solution [17] by his own method which also does not reduce to the Schwarzschild form in the absence of the magnetic parameter. The elegance of our technique is that a stationary gravitational two-soliton solution directly goes over to a static magnetovac solution and vice versa by two simple transformations, and the solutions are obtained in a compact form.

For a particular value of the magnetic parameter $\alpha$ (i.e. $\alpha=$ 1), the mass of the source vanishes and one obtains the dipole moment of a massless source. This corresponds to a finite current loop at large distances. The magnetic dipole moment directly depends on the strength of the current loop. Thus the study on magnetic dipole moment bears some relevance for the analysis of real astrophysical objects which are assumed to be usually charged due to a significant charge separation during their evolution.
Acknowledgements One of the authors (S.C.) acknowledges the University Grants Commission, India, for sanctioning a research project (vide Research Project No. F.PSW-07/06-07 (ERO)).

Open Access This article is distributed under the terms of the Creative Commons Attribution 4.0 International License (http://creativecomm ons.org/licenses/by/4.0/), which permits unrestricted use, distribution, and reproduction in any medium, provided you give appropriate credit to the original author(s) and the source, provide a link to the Creative Commons license, and indicate if changes were made. Funded by SCOAP ${ }^{3}$.

\section{References}

1. V.A. Belinskii, V.E. Zakharov, Sov. Phys. JETP 48, 985 (1978)

2. V.A. Belinskii, V.E. Zakharov, Sov. Phys. JETP 50, 1 (1979)

3. K. Schwarzschild, Berl. Akad. Ber. 7, 189 (1916)

4. R.P. Kerr, Phys. Rev. Lett. 11, 237 (1963)

5. E.T. Newman, T. Unti, L. Tamburino, J. Math. Phys. 4, 915 (1963)

6. K.C. Das, S. Chaudhuri, Pramana J. Phys. 40, 277 (1993)

7. S. Chaudhuri, Pramana J. Phys. 58, 449 (2002)

8. P.S. Letelier, Phys. Rev. D 20, 3728 (1982)

9. P.S. Letelier, J. Math. Phys. 26, 467 (1985)

10. E. Verdaguer, J. Phys. A Math. Gen. 15, 1261 (1982)

11. J. Carot, E. Verdaguer, Class. Quantum Gravity 6, 845 (1989)

12. S. Chaudhuri, K.C. Das, Gen. Relativ. Gravit. 29, 75 (1997)

13. D. Kramer et al., Exact Solutions of Einstein' Field Equations, 2nd edn. (Cambridge University Press, Cambridge, 2003)

14. T.I. Gutsunaev, V.S. Manko, Phys. Lett. A 132, 85 (1988)

15. K.C. Das, S. Banerji, Gen. Relativ. Gravit. 9, 85 (1978)

16. K.C. Das, J. Phys. A 13, 2985 (1980)

17. W.B. Bonnor, Z. Phys. 190, 444 (1966) 